\title{
Design and Manufacturing Main Linac Superconducting Quadrupole for ILC at Fermilab
}

\author{
Vladimir S. Kashikhin, Nikolai Andreev, Vadim V. Kashikhin, Michael J. Lamm, Mauricio L. Lopes, \\ John C. Tompkins, Alexander V. Zlobin
}

\begin{abstract}
The design and manufacturing of the first model of an International Linear Collider (ILC) Main Linac superconducting quadrupole is in progress at Fermilab. The quadrupole has a $78 \mathrm{~mm}$ aperture, a $36 \mathrm{~T}$ integrated gradient, and a cold mass length of $700 \mathrm{~mm}$. A superferric magnet configuration with iron poles and four racetrack coils was chosen based on magnet performance, cost, and reliability considerations. Each coil is wound using enamel insulated, 0.5 mm diameter, NbTi superconductor. The quadrupole package also includes shell type dipole steering coils. The results of the quadrupole design, including magnetic and mechanical analyses, are presented. Specific issues related to the quadrupole magnetic center stability, superconductor magnetization and mechanical stability are discussed and analyzed. The magnet quench protection system, current leads, and mounting the quadrupole inside ILC Main Linac cryomodule will also be briefly discussed.
\end{abstract}

Index Terms-Collider, Magnetic Design, Main Linac, Manufacturing, Superconducting Quadrupole.

\section{INTRODUCTION}

$\mathrm{T}$ HE Main Linac Superconducting Quadrupole for the future International Linear Collider [1] is now under design at Fermilab. Several superconducting quadrupole magnet models with similar parameters [2-4] have been designed and built for the LHC, the TESLA Test Facility, and XFEL. The main direction of this activity was to choose a magnetic configuration, a magnet manufacturing technology and reach the required field integrated gradient.

This paper describes the magnet design and performance issues: the quadrupole magnetic center stability, effects caused by superconductor magnetization, a comparison of shell type and racetrack type dipole correctors inside the quadrupole package, and coupling effects between the quadrupole and the dipole correctors during Beam Base Alignment (BBA) procedures. Some of these issues were investigated for LHC correctors [5-6] but for ILC quadrupoles, the requirement on magnetic center stability is an order of magnitude more stringent.

Manuscript received August 27, 2007. This work was supported by the U.S. Department of Energy.

V. S. Kashikhin, N. Andreev, M. J. Lamm, M. L. Lopes J. C. Tompkins, A. V. Zlobin are with the Fermi National Accelerator Laboratory, Batavia, IL 60510 USA (corresponding author phone: 630-840-2899; fax: 630-840-3369; e-mail: kash@fnal.gov).

\section{QUADRUPOLE SPECIFICATION}

The Main Linac quadrupole specifications were defined during the ILC Reference Design Report study [1]. The quadrupole is mounted in the center of the cryomodule, occupying the space of one RF cavity, as shown in Fig. 1. Table I shows the general specified quadrupole parameters.

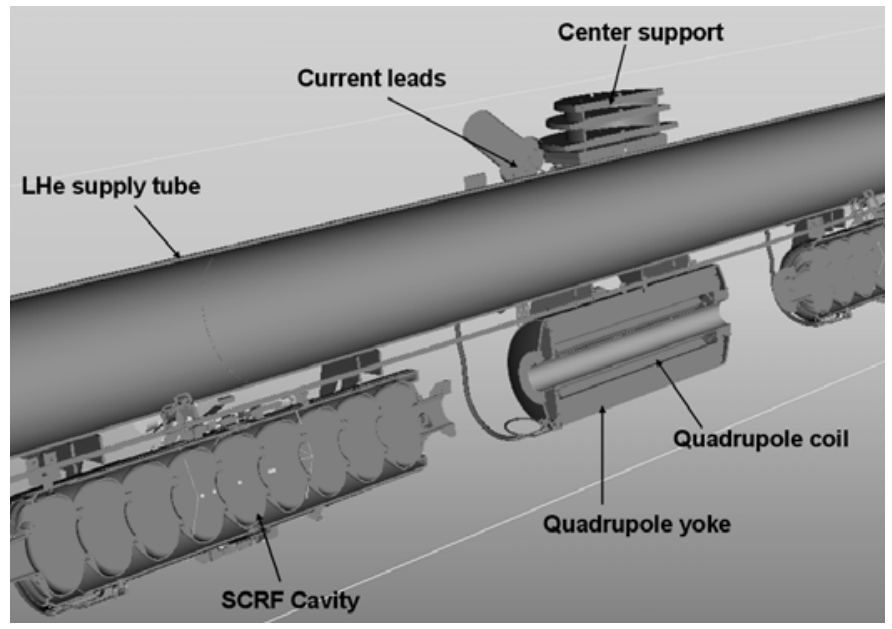

Fig. 1. Quadrupole inside cryomodule.

TABLE 1 Quadrupole Specification

\begin{tabular}{ccc}
\hline \hline Parameter & Unit & Value \\
\hline Integrated gradient & $\mathrm{T}$ & 36 \\
Aperture & $\mathrm{mm}$ & 78 \\
Effective length & $\mathrm{mm}$ & 660 \\
Peak gradient & $\mathrm{T} / \mathrm{m}$ & 54 \\
Field non-linearity at 5 mm radius & $\%$ & 0.05 \\
Dipole trim coils integrated strength & $\mathrm{T}-\mathrm{m}$ & 0.075 \\
Quadrupole strength adjustment for BBA & $\%$ & -20 \\
Magnetic center stability at BBA & $\mathrm{micron}$ & 5 \\
Magnetic center offset in cryomodule & $\mathrm{mm}$ & 0.3 \\
Quadrupole azimuthal offset in cryomodule & $\mathrm{mrad}$ & 0.3 \\
Liquid helium temperature & $\mathrm{K}$ & 2 \\
Quantity required & & 560 \\
\hline \hline
\end{tabular}


The quadrupole strength will be varied up to $20 \%$ (decrease) during BBA procedures. If the beam is offset relative to the magnetic center it will be displaced as the quadrupole strength changes. This offset and the corresponding beam deflection will be monitored by a Beam Position Monitor (BPM) with submicron accuracy mounted adjacent to the quadrupole assembly. These data will determine currents in the vertical and horizontal steering dipoles to match the quadrupole and the beam centers. This procedure requires the quadrupole magnetic center to be stable at the $\leq 5$ micron level, independent of the gradient setting, during all accelerator operations. This is a challenging requirement and it will require significant $R \& D$ to qualify any proposed magnet design and its integration with the cryomodule.

\section{QuadRupole Package Design}

In general there are two options for the magnet design. The first is a shell type design [2], [3] and the second is a "superferric" design [4], [5] where saturated iron poles form a substantial part of magnetic field in the quadrupole aperture. The second version was chosen as more promising for magnetic center stability, ease of manufacturing lower cost. Fig. 2 shows the quadrupole cross-section view.

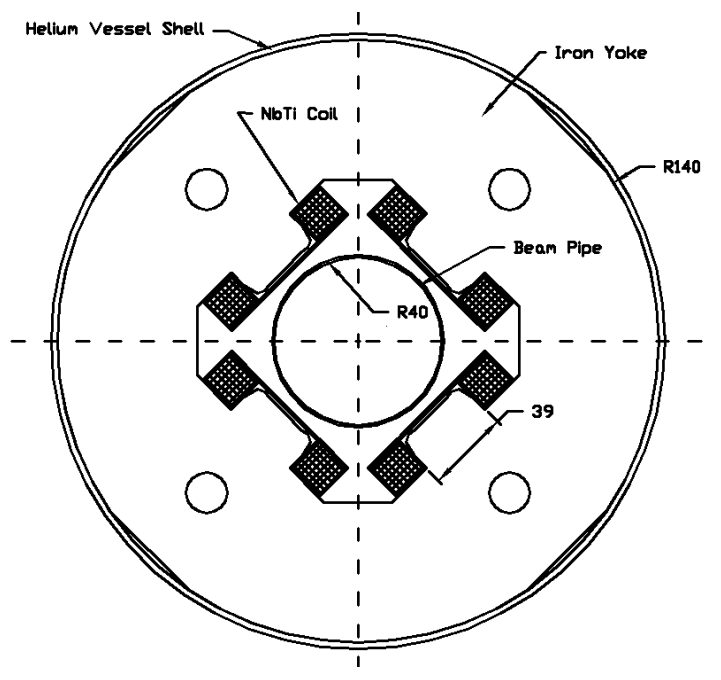

Fig. 2. Quadrupole cross-section.

TABLE II Quadrupole Parameters

\begin{tabular}{ccc}
\hline \hline Parameter & Unit & Value \\
\hline Peak current at 36 T gradient & $\mathrm{A}$ & 100 \\
Magnet length & $\mathrm{mm}$ & 680 \\
NbTi superconductor diameter & $\mathrm{mm}$ & 0.5 \\
Superconductor filament size & $\mu \mathrm{m}$ & 3.7 \\
Superconductor critical current at 5 T and & $\mathrm{A}$ & 200 \\
4.2 K & & \\
Coil maximum field & $\mathrm{T}$ & 3.3 \\
Coil number of turns & & 800 \\
Yoke outer diameter & $\mathrm{mm}$ & 280 \\
\hline \hline
\end{tabular}

\section{A. Quadrupole Magnetic Design}

The proposed quadrupole has a simple configuration where the magnetic field is generated by four racetrack type coils. Field quality is not a significant issue for this magnet because the good field region is only out to a $5 \mathrm{~mm}$ radius and the positions of the iron poles and coils have relatively small influence. The, iron poles, even when saturated, increase the quadrupole strength. The magnet cross-section was optimized initially with OPERA 2D and 3D [7]. Fig. 3 shows the model and flux distribution.

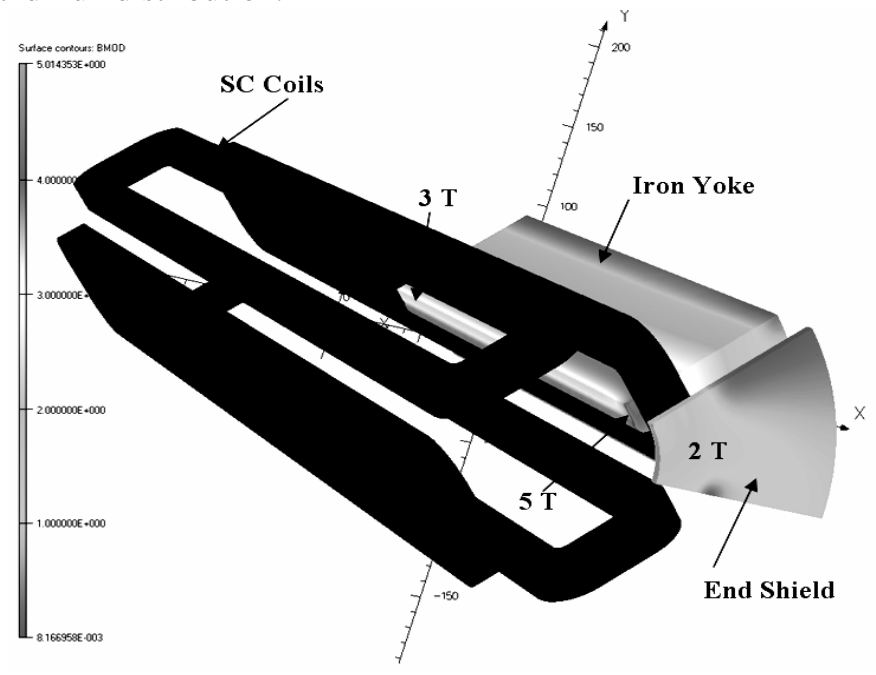

Fig. 3. Model geometry and iron flux density.

The field at the pole is $3 \mathrm{~T}$ in the center and $5 \mathrm{~T}$ in the pole ends. The integrated field homogeneity at various specified reference radii is shown in Fig. 4.

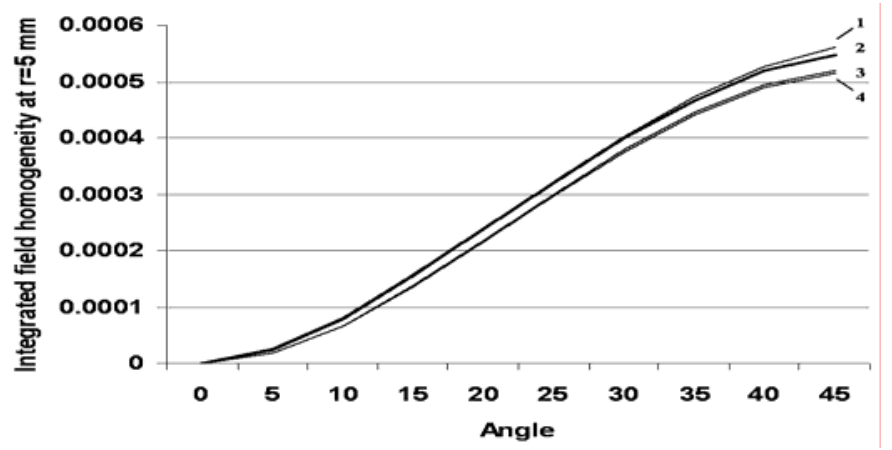

Fig. 4. Quadrupole integrated field homogeneity at $1-2.2$ A, $2-45$ A, $3-$ $89 \mathrm{~A}$, and 4 -100 A currents.

The maximum value $\sim 0.05 \%$ is the total sum of all allowed harmonics and meets the specifications. Further geometric optimization with pole holes could improve this result. As follows from Fig. 4 there is less than one unit of field distortion caused by iron saturation effects in pole area.

The quadrupole is mounted close (see Fig. 1) to the SCRF cavities. Fringing magnetic fields adjacent to an SCRF area must be less than $1 \mu \mathrm{T}$ during cavity cool down to avoid trapping flux in the $\mathrm{Nb}$ superconductor and lower than $10 \mu \mathrm{T}$ during cavity operation [2]. The fringing field from the quadrupole is reduced by iron end plates. The magnetic field outside quadrupole decays from 240 Gauss to less than 1 Gauss at distance $60 \mathrm{~mm}$ along $\mathrm{Z}$ - axis. It is amused that the 
SCRF will have a ferromagnetic shield with efficient shielding of external fields of up to several Gauss.

\section{B. Dipole Correctors}

The quadrupole package should have dipole correction coils to provide very accurate $(\sim 5 \mu \mathrm{m})$ matching of the quadrupole magnetic center with the center of electron beam. Two options for the dipole correctors were investigated. The first was shell type coils placed on the outer surface of the beam tube, while the second option used dipole coils wound on the the outer surfaces of the quadrupole racetrack coils and connected so as to generate vertical and horizontal dipole fields.

As mentioned previously, accurate beam and quadrupole center matching is a part of Beam Base Alignment (BBA) procedure. The quadrupole strength is reduced by $20 \%$ and if there is an offset, the Beam Position Monitors (BPM) attached to each quadrupole will determine it and a corresponding signal will drive the current in the dipole correctors to correct this offset. The quadrupole magnetic center should be stable to within several microns accuracy during the BBA procedure. One of the possible reasons for a shift in the quadrupole center could be the influence of superconductor magnetization effects. Fig. 5 shows the quadrupole center displacement due to superconductor magnetization for shell type dipole coils at zero current in the corrector coils.

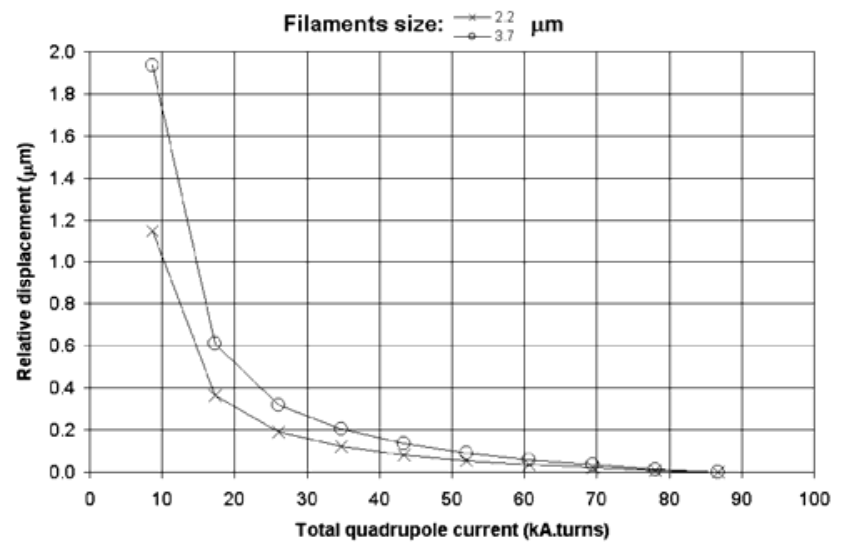

Fig. 5 Quadrupole center displacement for different currents and zero dipole fields.

For racetrack coils, the center is stable due to the quadrupole-like symmetry of the coils. For the shell-type at low quadrupole strength, the center is shifted in about 2 microns (for a filament size of 3.7 microns). The situation is more complicated when the quadrupole field is combined with a strong dipole field.

Fig. 6 shows the center displacement for three different quadrupole fields when the magnet is cycled between 80 to $100 \%$ (the corrector coils are not powered). At low quadrupole gradient $(9.2 \mathrm{~T} / \mathrm{m})$, the magnetic center displacement could be $\sim 5 \mu \mathrm{m}$. These effects could be further reduced by using superconductor with smaller diameter filaments. Another approach is to move the dipole correctors to a separate unit.

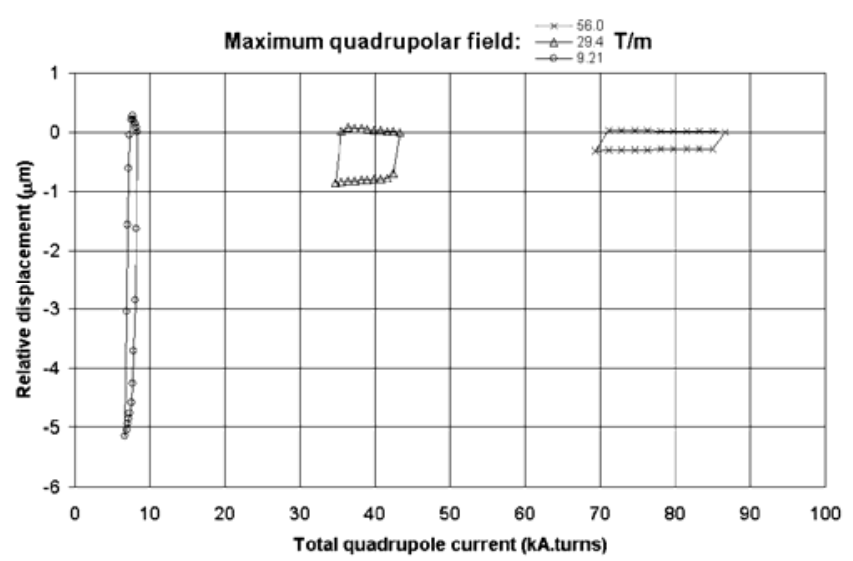

Fig. 6 Quadrupole center displacement at BBA for different quadrupole currents.

It should be noted that the dipole corrector strength changes at different quadrupole gradients because of iron yoke saturation effects and proper power supplies programming needed for BBA procedure.

\section{Mechanical Design}

The compact $20 \mathrm{~mm} \times 20 \mathrm{~mm}$ superconducting coils carrying the maximum current $100 \mathrm{~A}$ are loaded by large Lorentz forces: $\mathrm{Fx}=133 \mathrm{kN} / \mathrm{m}, \mathrm{Fy}=-34.4 \mathrm{kN} / \mathrm{m}$. To protect the coil from motion and provide better mechanical stability they are wound into racetrack type stainless steel channels. Both sides of the coils are supported by steel plates which go along pole tip surfaces. It makes the coil structure more rigid in the transverse direction. Fig. 7 shows the main stresses in the coil at maximum current.

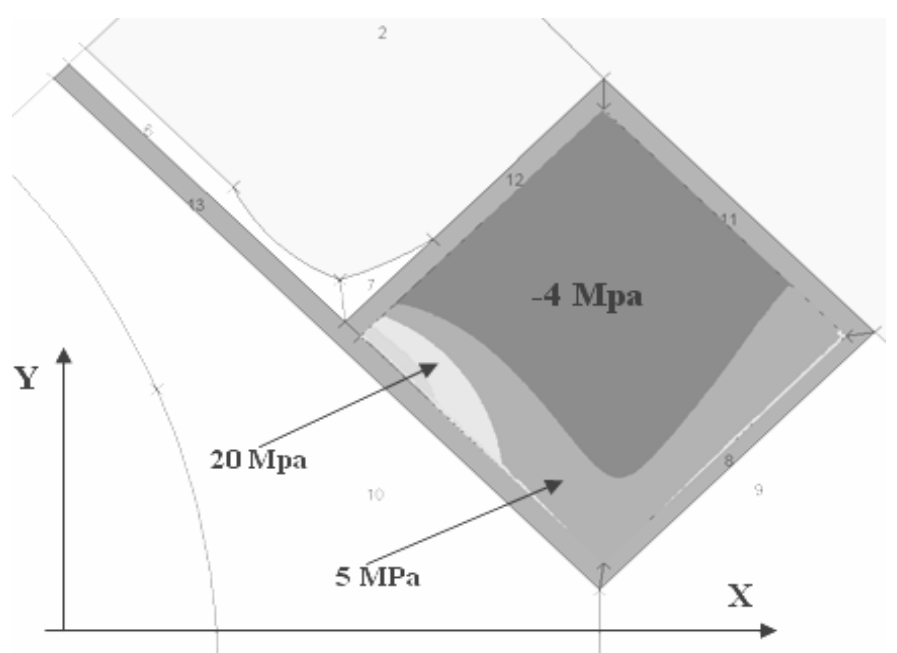

Fig. 7. Quadrupole coil principal stress distribution at maximum current.

The maximum stress in the coil corner is less than $50 \mathrm{MPa}$ and in the steel channel, no more than $120 \mathrm{MPa}$. The coil movement is about 11 microns with respect to the pole. The magnetic field simulations showed $\sim 2$ microns the quadrupole magnetic center shift when only one coil side moves from pole on distance 10 microns. There are two options to limit this movement: provide coil support on the outer surfaces using mid-plane wedges inserted between neighboring coils or use aluminum channels which will provide pre-stress during cool 
down due to greater thermal contraction and will protect the coils from pole separation. Both options will be investigated during model magnetic center stability tests.

\section{Quench Protection}

The quadrupole has a large number of turns, high inductance and $40 \mathrm{~kJ}$ of stored energy in the magnetic field. An active quench protection system will be used where a quench will detected by voltage taps, which initiates coil heaters and inserts an external dump resistor into the circuit for energy dissipation. Fig. 8 shows how the magnet parameters change in time during the quench calculated by ROXIE [8].

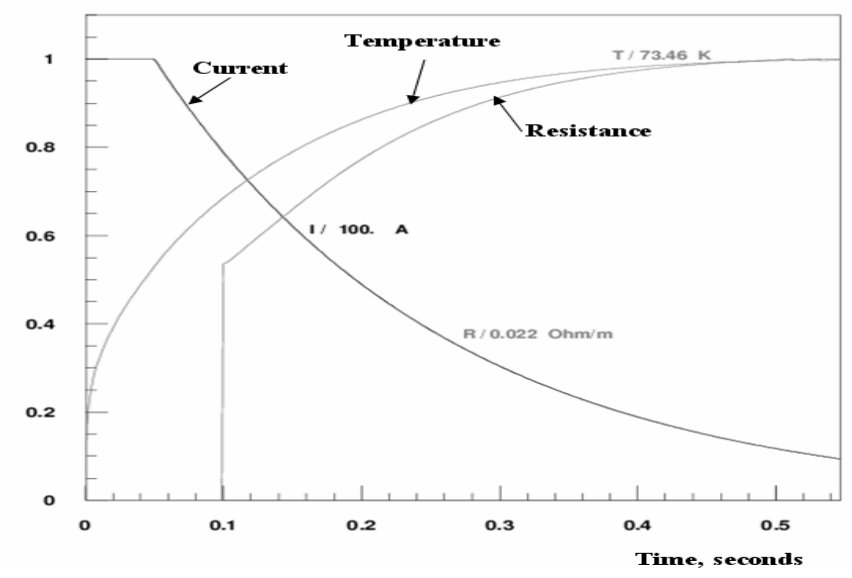

Fig. 8. Quadrupole superconducting coil parameters during quench.

A quench detected after $50 \mathrm{~ms}$, with a heater response delay time of $100 \mathrm{~ms}$, will result in an acceptable $74 \mathrm{~K}$ rise in the superconducting coil temperature. The maximum voltage will not exceed $1 \mathrm{kV}$, limited by the $10 \mathrm{Ohm}$ external dump resistor.

\section{Magnet Manufacturing}

Several technical decisions were chosen to simplify magnet manufacturing:

- Superferric magnet configuration;

- Racetrack coils;

- $\quad$ Single wire winding technique;

- Coils wound into stainless steel channels which are used as winding mandrels and as closed molds for epoxy vacuum impregnation;

- Laminated iron yoke with a single lamination used for cross-section (i.e., not 4 pieces);

- Coils are assembled with yoke through the magnet aperture and bolted to the yoke at the ends.

Fig. 9 shows a racetrack coil after winding. During coil winding, large pressure accumulated on the kapton ground insulation at small radii at the coil ends; a proper thickness of insulation is needed in these areas. The iron yoke will be assembled from laser cut laminations: AISI 1006 low carbon steel of $1.5 \mathrm{~mm}$ thickness is being used. The overall accuracy of the measured laminations is in the range of $50 \mu \mathrm{m}$ and in the pole tip areas $25 \mu \mathrm{m}$. The quadrupole manufacturing is in progress now.

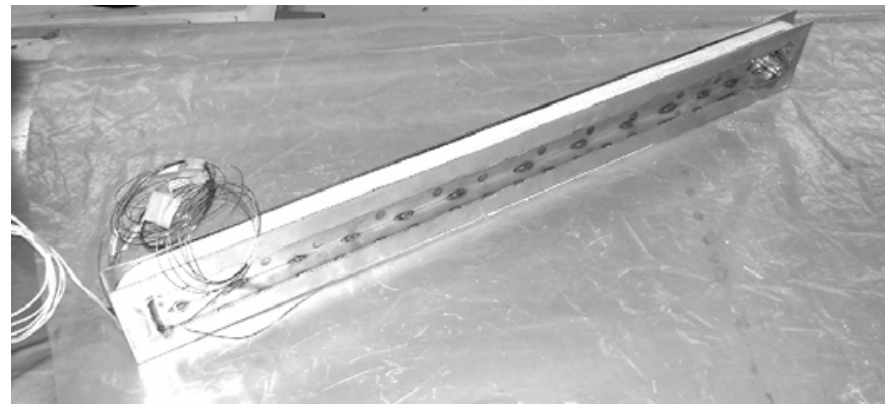

Fig. 9. Quadrupole racetrack coil.

\section{CONCLUSION}

The proposed magnet configuration is a very promising for further investigations. Careful tests and magnetic center position measurements should verify this approach. Nevertheless, it is possible to highlight the following issues and solutions discussed in the paper:

- Superconductor magnetization at small filament size effects the magnetic center position at the level of several microns;

- There is a strong coupling between the quadrupole and dipole correctors through iron yoke saturation;

- Racetrack coils in channels provide a strong and robust mechanical structure;

- The Magnet requires an active quench protection system;

- End plates are used to provide effective end field shielding;

- Shell type correctors generate larger fields than racetracks; but racetrack dipole correctors have lower superconductor magnetization effects;

- Moving the dipole correctors from the quadrupole will eliminate coupling and simplify the BBA procedure.

The first quadrupole package will be tested in the fall of 2007.

\section{REFERENCES}

[1] "International Linear Collider Reference Design Report", http://www.linearcollider.org/cms/?pid=1000025.

[2] “TESLA Design Report”, Hamburg, DESY 2001-11, 2001.

[3] A. Ijspeert, et. al., "Principles developed for the construction of the high performance, low-cost superconducting LHC corrector magnets", IEEE Transactions on Applied Superconductivity, vol. 12, No. 1, March 2002, pp. 90-93.

[4] A. Koski, R. Bandelmann, S. Wolff, "Superconducting magnet package for the TESLA test facility", IEEE Transactions on Magnetics, vol. 32, No. 4, July 1996, pp. 2155-2158.

[4] F. Toral, et. al., "Design and fabrication study on the TESLA500 superconducting magnets", IEEE Transactions on Applied Superconductivity, vol. 12, No. 1, March 2002, pp. 282-286.

[5] W. Venturini Delsorado, M. Karppinen, "Cold test results of the inner triplet orbit correctors for the LHC", IEEE Transactions on Applied Superconductivity, vol. 16, No. 2, June 2006, pp. 277-280.

[6] L. Bottura, et. al., "Magnetic alignment measurements at room and cryogenic temperature of LHC cryodipoles and associated correctors at CERN", IEEE Transactions on Applied Superconductivity, vol. 14, No. 2, June 2004, pp. 227-230.

[7] OPERA 2D and TOSCA, Vector Fields Ltd., 24 Bankside, Kidlington, Oxford OX5 1JE, England.

[8] S. Russeschuk, "Electromagnetic design and mathematical optimization methods in magnet technology", CERN AT-MEL-EM, version 3.1, January 2006, p. 595. 